\title{
Review Article \\ Comprehensive Quality Control of the Regenerative Therapy Using Platelet Concentrates: The Current Situation and Prospects in Japan
}

\author{
Tomoyuki Kawase ${ }^{1}{ }^{1}$ and Kazuhiro Okuda ${ }^{2}$ \\ ${ }^{1}$ Division of Oral Bioengineering, Institute of Medicine and Dentistry, Niigata University, Niigata 951-8514, Japan \\ ${ }^{2}$ Division of Periodontology, Institute of Medicine and Dentistry, Niigata University, Niigata 951-8514, Japan \\ Correspondence should be addressed to Tomoyuki Kawase; kawase@dent.niigata-u.ac.jp
}

Received 15 January 2018; Accepted 2 April 2018; Published 21 May 2018

Academic Editor: Elena Jones

Copyright (C) 2018 Tomoyuki Kawase and Kazuhiro Okuda. This is an open access article distributed under the Creative Commons Attribution License, which permits unrestricted use, distribution, and reproduction in any medium, provided the original work is properly cited.

\begin{abstract}
Platelet concentrates (PCs), represented by platelet-rich plasma (PRP), have been widely applied in the fields of regenerative and aesthetic therapies. PCs' mechanisms of action, however, are too complicated, and it is not easy to present the whole picture; besides, clinical outcomes are hardly reproducible in many cases. Therefore, several medically advanced countries seemingly intend to regulate PC therapies weakly or strictly because of the increasing popularity. Japan established laws and regulations for PC therapy in the "Act on the Safety of Regenerative Medicine" along with the "Pharmaceuticals, Medical Devices and Other Therapeutic Products Act" in 2014, which, to our knowledge, represent the strictest regulatory framework for production and therapeutic use of PCs in the world. According to these laws and regulations, PCs produced for topical use should be prepared as cellbased medicinal products, essentially as should stem cells, in accordance with their registered ("licensed" under actual conditions) standard operating procedures. Nonetheless, criteria for their quality are not standardized. In this review, we discuss the quality of PC preparations by focusing on the basic concept and regulatory framework of regenerative medicine in Japan. Within the new framework, PC therapy is regulated by a specific notification and registration system, as is stem cell therapy. In comparison with the latter, however, risk factors that hamper successful PC therapy are much fewer. Via appropriate evaluation of patients' conditions and whole-blood samples by simple and sensitive but not yet fully standardized assays, it is theoretically possible that PC quality will be controlled nearly completely. In addition to or instead of standardization of preparation protocols, standardization of preoperative examination of individual PC preparations is an urgent task for improving and guaranteeing the safety and efficacy of PC therapy.
\end{abstract}

\section{Introduction}

Regenerative therapy using platelet concentrates (PCs), such as platelet-rich plasma (PRP), is an adjuvant biological therapy $[1,2]$. Despite more than 2 decades of use of PCs in various areas of regenerative medicine, the essence of PC therapy seems not to be regarded correctly by many clinicians: basically, the mechanisms of its action are not yet fully clarified and wide variations of its clinical outcomes remain to be clearly explained. Partially for these reasons, some regulatory authorities in individual countries are seemingly intending to regulate medical use of PCs by establishing a new regulatory framework. In this review, we introduce the basic concept of the regulatory frameworks for cell-based medicinal products (CBMPs) in Japan, analyze the current situation and understanding of PC therapy, and finally propose the Must-Do list for improving the quality of PC therapy.

\section{The World Is Changing}

2.1. Changes in the United States and Europe. The Food and Drug Administration (FDA) of the United States has not yet attempted to regulate but raised some concerns recently over activated PRP [3]. In Europe, the regulatory landscape (related to the products derived from the manipulation of whole blood), which sets forth quality and safety rules for 
TABLE 1: Classification of cell-based therapies by their potential risks.

\begin{tabular}{lcc}
\hline Class & Definition & Example \\
\hline 1st & High-risk therapy involving cells with little or no & ES cells, iPS cells \\
& supporting clinical evidence & Sematic stem cells \\
2nd & Ledium-risk therapy involving cells currently being & implemented in clinical settings \\
& Low-risk therapy involving cells that are subjected to & Somatic cells \\
& limited manipulation or processing &
\end{tabular}

This table was translated from the original presented in the ministry's guidance document [9]. ES cells: embryonic stem cells. iPS cells: induced pluripotent stem cells.

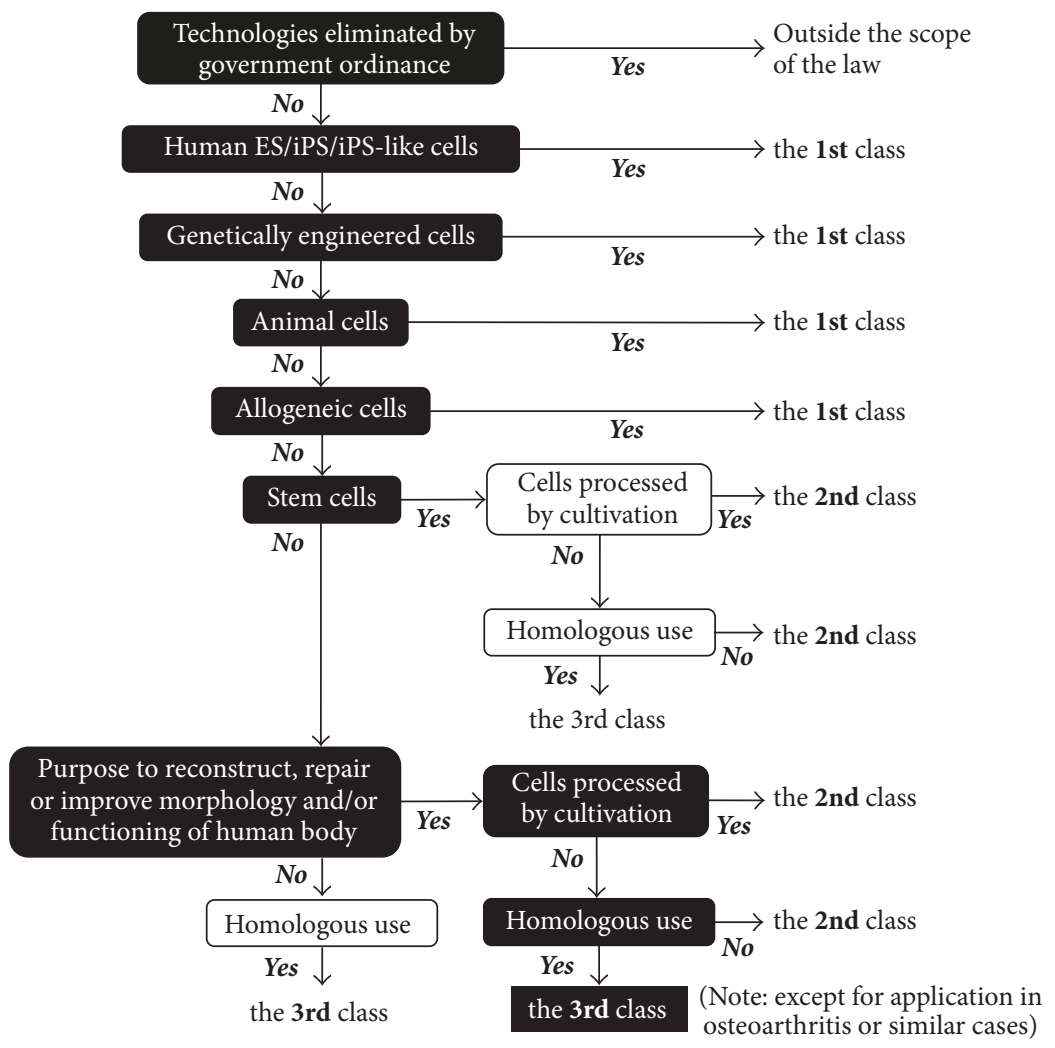

FIGURE 1: Classification of PC therapies in accordance with the risk tree presented by the Ministry of Health, Labour and Welfare of Japan.

collecting, controlling, processing, preserving, and distributing human blood and its components, is currently governed by Directive 2002/98/EC of the European Parliament and Council of January 27, 2003, and is acknowledged in the various States of the Union with internal regulations [4]. In 2013, the Spanish Agency of Medicines and Medical Devices drew up a comprehensive report and resolution that for the first time regulates the use of PRP as a medicinal product for humans to adapt the applications of PRP to the new requirements of safety and efficacy $[5,6]$.

2.2. Changes in Japan. For maintenance and improvement of health of aged individuals, a new regulatory framework has been applied to regenerative medicine, and, for this purpose, two laws known as the "Act on the Safety of Regenerative Medicine" and the "Pharmaceuticals, Medical Devices and Other Therapeutic Products Act" came into effect on November 25, 2014 [7-9]. On the basis of the former law, the Ministry of Health, Labour and Welfare of Japan (MHLW) established a regulatory framework necessary for examination of the regenerative-medicine provision plan and for inspection of cell culturing and processing facilities, aiming to ensure full safety of regenerative medicine and related modalities and to promote the development of its practical applications [8]. In these regulations, MHLW classifies cell-based therapy into three categories (Table 1); however, medical technologies specified by the government ordinance, that is, blood transfusion, hematopoietic stem cell transplantation, and assisted reproduction, are excluded from these regulations. Their principles and essence have been described and discussed in detail in several articles [7, 10, 11]; however, no convincing legal basis for classification of PCs has been provided. According to the risk tree presented by MHLW, we can recognize that regenerative and aesthetic therapies using PCs are categorized into "the 3rd class" under the regulations (Figure 1). Please check a series of criteria 
written in white inside black boxes to follow the classification process of PC therapy. To our knowledge, these regulations are the strictest for the use of PCs in regenerative and aesthetic therapies among medically advanced countries. Although it is hard to predict how many regulatory authorities in individual countries will follow Japan's actions, it would be beneficial for patients to improve the clinicians' knowledge and awareness of the safety of "home-made biologicals" and ways to minimize possible adverse effects and complications.

Here, the definition of "processing" should be mentioned: this term is defined as procedures related to artificial cell growth, differentiation, immortalization, and activation, among other methods. Nonetheless, the simplest procedures, such as separation and mincing of tissues and/or cells (note: chemical-reagent-dependent separation techniques are excluded), treatment with antibiotics, $\gamma$-ray irradiation, freezing, and thawing, shall not be deemed "processing" as defined by these regulations. Nevertheless, when cells are processed to have a structure and function that are different from their original ones regardless of the criteria described above, this procedure shall be deemed "processing" as defined by these regulations. Preparation of PCs always includes centrifugation and sometimes anticoagulants and coagulation factors. Although this procedure of course is not aimed at forcing platelets and leukocytes to grow, differentiate, or get immortalized, platelets can be more or less activated by centrifugation and coagulation factors. In public interpretation, the crucial criterion for PCs is probably "the purpose of processing," that is, the purpose of inducing expression of supraphysiological structure and function of platelets.

It was not and is not easy for many clinicians to accept and learn this new regulatory framework because they have never been educated or trained to understand the basic concepts of CBMPs, good manufacturing practice (GMP), and/or standard operating procedure- (SOP-) based preparation and treatment. On the other hand, we have to accept that the world is changing. In general, clinicians' discretion tends to be more restricted now than before, and their decisions now tend to be controlled in detail by specific regulatory arrangements, especially in the field of advanced medical care, including cell-based therapy.

\section{Quality of PCs}

3.1. Quality of CBMPs: A General Concept. In general, CBMPs should be prepared at licensed facilities in accordance with the licensed SOPs based on the concepts of GMP. Haddock et al. [13] mentioned that determining a specific strategy for the creation of a GMP facility for cell manufacturing will depend largely on the appropriate business model and the type of cell product. Whether a bedside (i.e., chairside in dentistry) point-of-care manufacturing model (e.g., for autologous cells), a regional manufacturing hub model, or a centralized manufacturing model is the most appropriate must be decided a priori based on patients' needs, transportation and storage options, cost, and flexibility of the product. In addition to clinical manufacturing facilities, there is a great need for GMP (or at least, GMP-like) facilities for product and process verification and validation as well as workflow simulation. In the current situation, major PC preparations are regarded as products generated by means of a point-ofcare manufacturing model without transportation or storage. By contrast, with changes in patients' and/or clinicians' needs in the near future, this manufacturing style may change to the regional manufacturing hub model or something similar. In any case, each patient's CBMPs should be considered one batch, and quality of each batch should be ensured before shipping; this approach is necessary to meet current GMP (cGMP) requirements and to get PCs integrated into safe and effective cell-based therapeutics [14].

3.2. What Is Included in the "Quality" of CBMPs. Quality is subdivided roughly into two closely related issues: safety and efficacy, which are further classified into sterility, purity, identity, potency, and stability $[15,16]$. We illustrated this concept with the flowchart of clinical trial in Figure 2. In clinical trial, safety and efficacy are examined successively at Phases I and II, respectively. Similarly, in quality assurance, safety is the main criterion for industrial products in the "broadest and the highest" concept, and efficacy is for conventional types of drugs in the "moderate" concept under the broadest concept. Purity, identity, and stability are also related to drug quality; however, in CBMPs, these criteria are closely related to tumorigenicity. Stem cells, which include pluripotent to multipotent stem cells processed for cell-based therapy, should essentially and ideally be examined prior to every shipping to a clinical organization or entity. When preoperative examinations cannot be performed in a timely manner, for example, in case of cell-specific problems, for example, chromosomal abnormality and tumorigenicity, only randomly selected cell product batches have been tested or the corresponding cell populations in parallel cultures have been evaluated after shipping. Nevertheless, this standardized examination procedure is intended for quality assurance of multipotent nucleated cells and may also be applied to other somatic cells. It is questionable from several biological standpoints (described in the next section) whether PCs should be regulated by the same criteria. According to the major differences between somatic stem cells and platelets or leukocytes in PC preparations (Table 2), platelets and leukocytes are clearly distinguishable from stem cells in the capacity for proliferation and differentiation.

3.3. General Quality of Prepared PCs. It has generally been accepted that quality - in a broad sense-of PC preparations is more or less influenced by devices employed and an operators' technique. The factors influencing PC quality are summarized in Table 3. When a needle is made of metal, of a narrow gauge, and/or long, platelets are likely to be easily activated to form aggregates in the absence of anticoagulants. A centrifugation protocol is well known to influence the quality of PC preparations in the absence of anticoagulants [17]. In particular, because contact of blood with the inner glass wall of a blood-collection tube triggers coagulation [18], the material of the tube and duration, force, and direction (angle) of contact severely affect the quality of platelet-rich fibrin (PRF) preparations. Furthermore, even though a blood-collection 

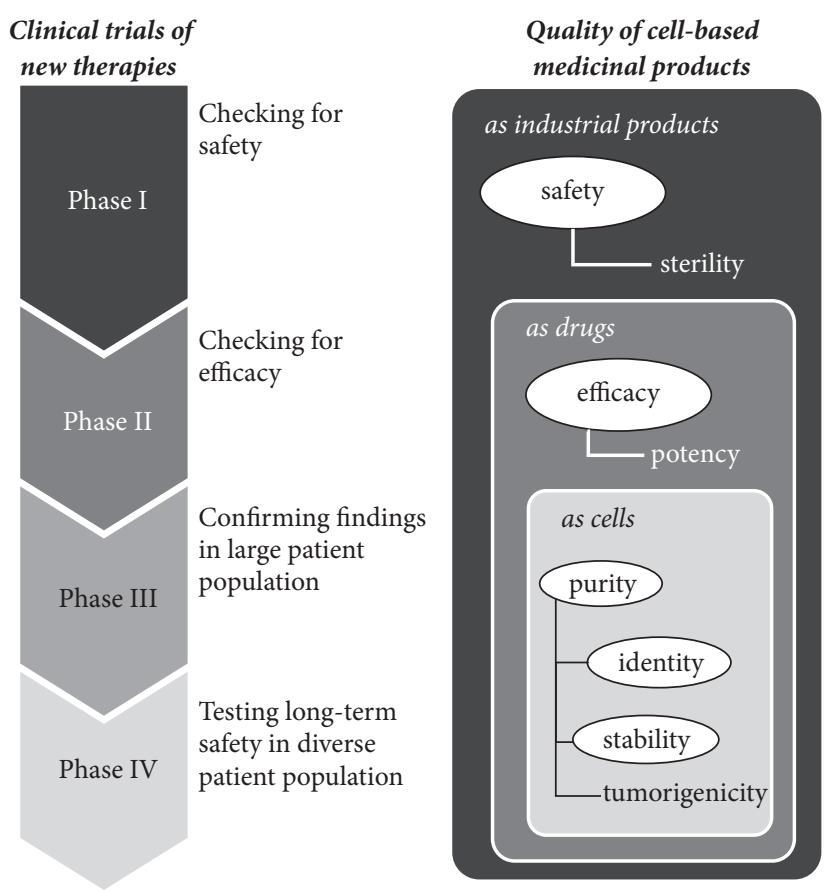

FIGURE 2: Illustration of general concept for quality of CBMPs and a flowchart of clinical trials for new therapies as reference.

tube is made of glass, it should be noted that the inner surface is usually coated with silicone or a similar polymer to improve biocompatibility. However, thickness and quality of this surface coating vary among individual products and individual lots of the same product depending on the proficiency of a manufacturer. In fact, we and other clinician groups have seen incomplete coagulation in imported offbrand glass tubes that are not licensed as a medical device in Japan. Additionally, blood-collection tubes are generally produced for laboratory experiments, not for preparation of implantable blood-derived materials. Therefore, clinicians should primarily employ tubes approved for preparation of PCs by authorized agencies of individual countries. If such products are not commercially available, clinicians should choose products manufactured in accordance with reliable quality systems of manufacturing, that is, by GMP facilities. For all countries around the world, especially for European countries, the CE mark may be a reliable standard of quality. Therefore, CE-labeled tubes are expected to function in the same way in the same products with respect to processing specifications or standards, thereby simplifying and favoring the use of PCs [4].

In addition, as for preparation of PRP from citrated whole blood, an operator's technique and choice of coagulation factors are highly important and influence quality. The use of ethylenediamine tetra-acetic acid (EDTA) in preparations of PRP is potentially more harmful, and large numbers of damaged platelets have been observed [19]. A trisodium citrate solution is an anticoagulant with no negative effects on PRP preparations and, consequently, acid-citrate-dextrose (ACD) is a preferred anticoagulant.
Poor pipetting skills may induce undesirable platelet activation and reduce platelet concentration, whereas added thrombin produces a fibrin matrix composed of relatively thin fibrin fibers [20].

3.4. Safety, Stability, Purity, Sterility, Identity, and Potency of $P C s$. In this subsection, we discuss these evaluation indicators for autologous PCs because allogeneic PCs are not generally accepted for regenerative therapy at present. In general, PCs are prepared from autologous whole-blood samples essentially in accordance with the licensed SOPs. Nevertheless, basically and theoretically, the safety of autologous PCs is supported by the following factors. First, autologous PCs are immunologically neutral and pose no danger of allergy, hypersensitivity, or foreign-body reactions. Second, because of autologous implantation, there are no worries about the transmission of infectious diseases, neoplastic cells, or other unknown factors. Third, regardless of origin, their major cell components are platelets and leukocytes: platelets are anucleate cells, whereas leukocytes, despite being nucleated cells, are highly differentiated cells and manifest no spontaneous growth activity (i.e., show high stability). Fourth, regardless of origin, PCs are subjected to only gravitybased fractionation and activation, which are considered minimal manipulations. Taken together with our clinical experience in the last 25 years in regenerative dentistry, the above observations suggest that the probability of either tumorigenicity, that is, strong instability, or other possible adverse effects can be ignored.

Despite being heterogeneous, that is, not purified, blood cell populations, as long as PCs are prepared aseptically, their 


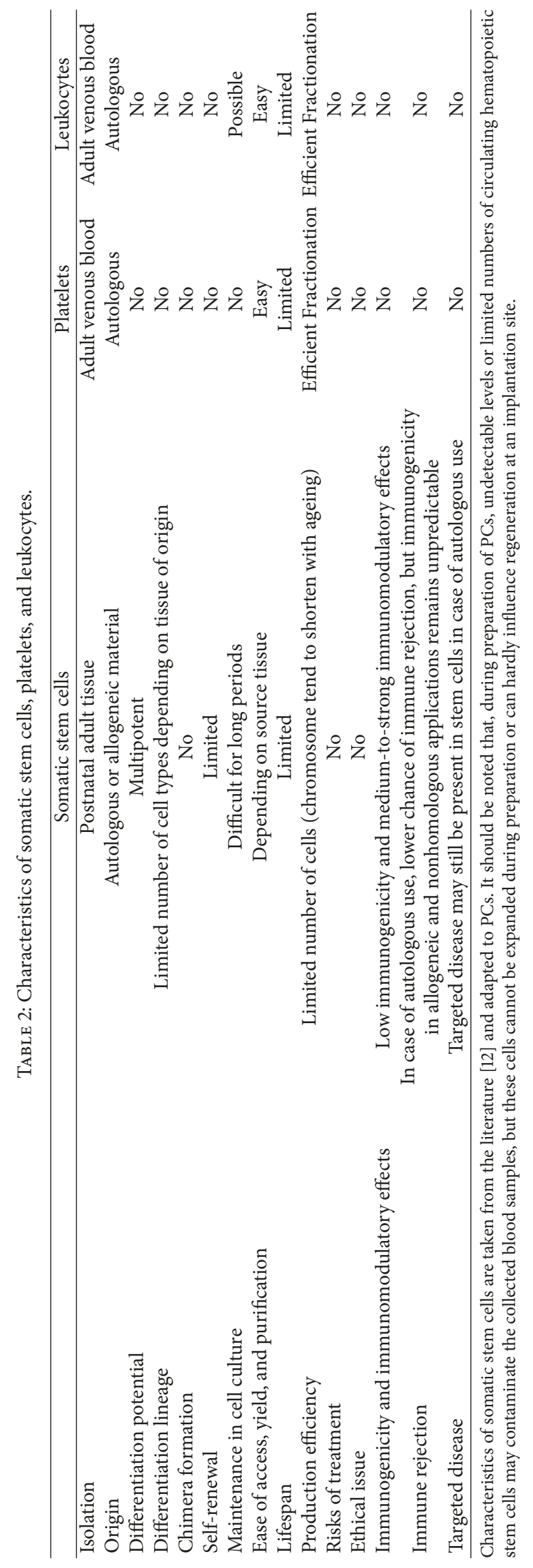


TABLE 3: Influential factors associated with quality of PCs.

\begin{tabular}{lcc}
\hline Preparation step & Influential factor & Details \\
\hline \multirow{2}{*}{ Blood collection } & Needle & Gauge, length, material, surface modification \\
& Tubing of butterfly needle & Diameter, length, material \\
& Syringe/tube & Material, surface modification \\
& Lag & Distance between blood-collection space and centrifuge \\
\hline \multirow{2}{*}{ Centrifugation } & Tube & Shape, material, surface modification \\
& Rotator & Swing or angle \\
Other handling & Centrifugal condition & Force, duration \\
& Pipetting & Technique, material
\end{tabular}

sterility and identity can be ensured. Their potency can be evaluated by several in vitro assays that do not require much time and is roughly proportional to the platelet count [21]. Therefore, point-of-care determination of platelet counts in prepared PCs is recommended as a minimum check point for efficacy assurance. If hematological analyzers are not available on site, just as bacterial counts, platelet counts in PRP can be conveniently and inexpensively determined by the spectrophotometric method at $615 \mathrm{~nm}$ (Kitamura et al., manuscript in submission). Furthermore, it is noteworthy that platelet counts in nonliquid products, such as PRF preparations, can also be determined accurately by our recently developed digestion method [22].

3.5. Systemic Conditions Influencing Quality of Whole-Blood Samples and Resulting PCs. PC therapy is widely employed as an adjuvant modality in different surgical procedures, more frequently in the areas of dentistry and orthopedics $[1,2,23]$. Therefore, variations in clinical cases and surgeon's techniques, in addition to those among individual samples, are considered more influential than variations in devices and tubes. Thus, as reported elsewhere [17, 24, 25], randomized controlled trials (RCTs) assessing limited numbers of clinical cases usually have low quality and cannot reach conclusive results on outcomes of PC therapy.

We propose that the general condition of a patient should be more carefully and precisely evaluated via his/her medical history and selected preoperative systemic analyses, especially blood tests. Blood coagulation is regulated mainly by the interactions of coagulation factors and platelets. Therefore, even though platelet counts may be substantially lower than the normal range, or platelet activity can be significantly suppressed, sufficient coagulation activity may compensate for these drawbacks. In this case, however, it is plausible that growth factors may not be concentrated at expected levels. In contrast, when coagulation activity is substantially lower than normal, compensation by platelets may be less adequate than expected.

These changes can often be induced by a disease, medication, mental or physiological stress, or other factors. Marques et al. [23] stated that there are some conditions where the indication must be followed with caution, as in cases of thrombocytopenia, platelet dysfunction syndrome, septicemia, hypofibrinogenemia, a recent febrile condition, anemia, cancer, skin lesions in the area of the injection, use of corticosteroids (up to 2 weeks before the procedure), nonsteroidal anti-inflammatory drugs (up to 48 hours before the procedure), or an active infection with Pseudomonas, Klebsiella, or Enterococcus.

To give examples that are more familiar to many clinicians, warfarin and aspirin reduce coagulation and platelet activities, respectively [26-29]. Type 2 diabetes mellitus is characterized by hemostasis dysfunction caused by endothelial anomalies and platelet hyperreactivity [30, 31]. Diet and nutrients have been demonstrated to influence platelet function [32, 33] although these alterations may usually be below severe pathological levels. In addition, mental or physical stress activates platelets to induce thrombosis [3438]. Possible effects of these systemic aberrations on quality of prepared PCs have not been clarified and need to be investigated to more precisely evaluate suitability of platelets and coagulation systems and to establish the cut-off values of several parameters to be inspected, such as a platelet count, platelet aggregation activity, prothrombin time, thrombin time, and coagulation time, for preparation of quality-assured PCs.

\section{General Risk Factors in Cell-Based Therapy and Possible Risks of PC Therapy}

As described in the earlier section, autologous PCs are immunologically neutral and carry no risk of transmission of microbial pathogens. Nonetheless, the efficacy of individual PC preparations is not always at or above standard levels that are sufficient for inducing tissue regeneration. In this section, we again analyze quality of PC therapy in terms of additional clinical criteria, risk factors, and risks. In comparison with stem cell therapy [12] and on the basis of risk factors associated with stem cell therapy, possible risks of PC therapy are listed in Table 4. The risk factors are categorized into three groups: intrinsic, extrinsic, and clinical factors. In any category, possible risks of PC therapy boil down to sterility and efficacy issues, which are considered minor risks compared to those of stem cell therapy. Therefore, also from the viewpoint of risk factors and risks, PC therapy can be regarded as the safest cell-based therapy. 


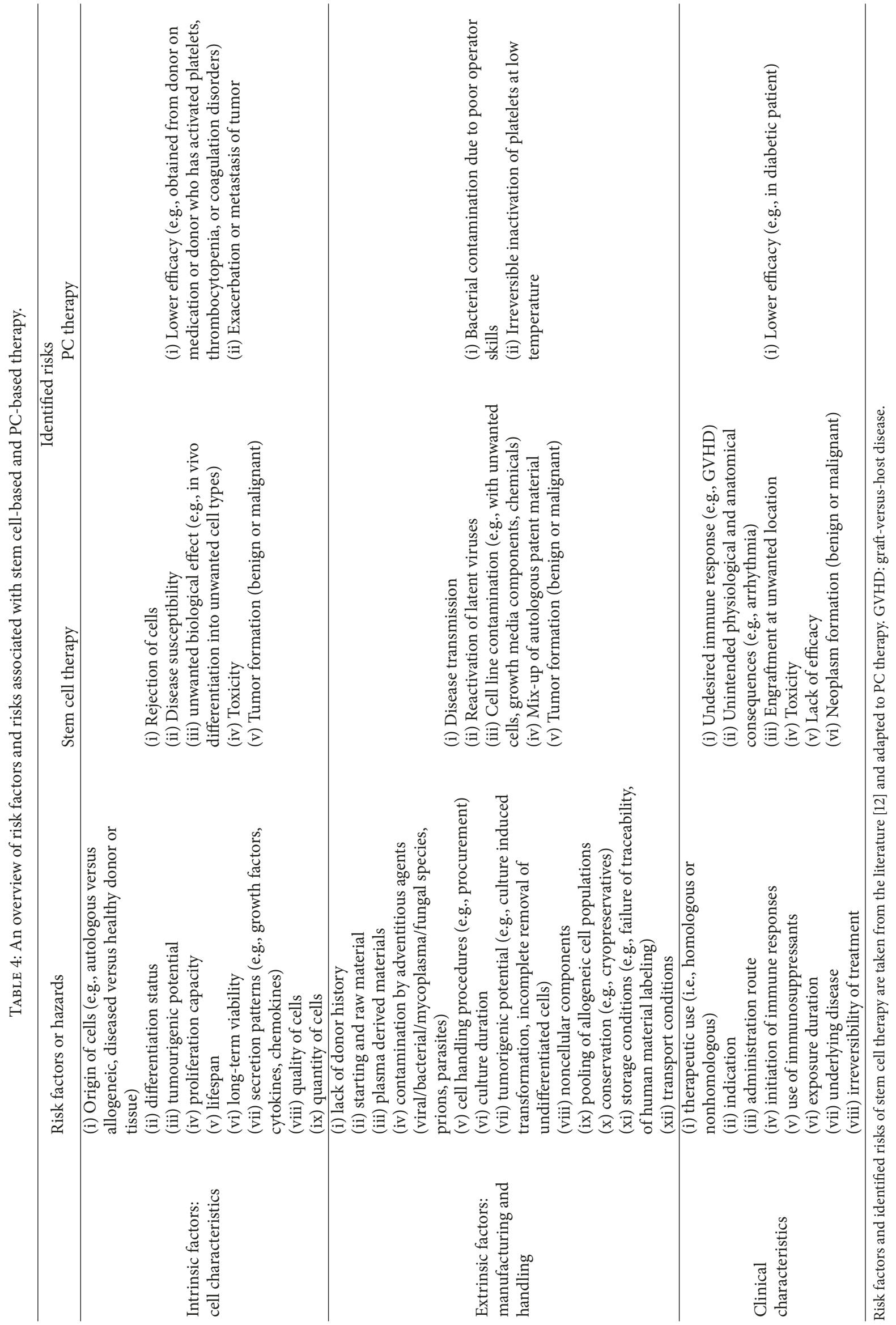




\section{Possible Case-Specific Risks Related to Contraindications}

Neither severe complications nor adverse effects of PC therapy have been reported in the past 25 years in the frontier field of regenerative medicine: regenerative dentistry. Consequently, there is a consensus that possible tumorigenicity or a severe complication can be ruled out; however, we are wondering whether those accumulated clinical data are sufficient to completely ignore any unknown hidden risks of PC therapy and to guarantee its safety for topical use. Here, we will voice several concerns. First, several in vitro studies have revealed that mitogenic action of leukocyte-rich PRP is not necessarily proportional to platelet counts and that an optimal concentration is approximately $2.5 \%$ in the culture medium or 2.5-fold above the basal levels [39, 40]. This result implies that an unidentified adverse factor(s), probably as well as inflammatory cytokines released by leukocytes, may act on cells nonspecifically and toxically in case of overdosing. Second, when a PC is combined with other biomaterials, for example, animal-derived, less characterized, but frequently applied bone substitutes, who can guarantee the safety of PC therapy? Is it possible to rule out the risk that PCs augment an unfavorable action of unidentified factors resulting from these biomaterials? This is off-label use of PCs. Third, why are the efficacy and clinical outcomes largely dependent on a host's topical and systemic conditions? That is the reason why PCs are considered adjuvant. Further basic studies are needed to clarify possible case-specific risks and disadvantages of PC therapy.

\section{Indications and Contraindications}

To administer a predictable or at least adequate PC therapy, it is important to have criteria for appropriate selection of patients and cases. In regenerative dentistry, relatively large alveolar bone defects may not be considered an appropriate indication for therapy by means of PC preparations alone. According to the currently accepted therapeutic strategy, it is better for clinicians to combine PCs with well-defined scaffolding materials and/or osteogenic cells for large bone defects [41, 42]. Even in case of small bone defects, if patients have a tumor in surrounding regions, it may be better to choose alternative therapeutic strategies rather than PC therapy. PC administration, as well as surgical stress, is thought to cause tumor tissues to grow faster because PCs contain various angiogenic factors [43]. In contrast, because of poor anatomical and physiological characteristics, tissue regions with weaker angiogenic properties, for example, a peri-implant region of gingival tissue and the relatively wide and flat region of the maxillary sinus, may not be suitable for successful regeneration by PC therapy [44].

As for systemic conditions, Sampson et al. mentioned in their article [45] that moderate contraindications of PC therapy include the presence of a tumor, metastatic disease, an active infection, or a platelet count $<10^{5} / \mu \mathrm{L}$ with hemoglobin $<10 \mathrm{~g} / \mathrm{dL}$. Pregnancy or active breastfeeding is contraindication. Patients with an allergy to bupivacaine
(Marcaine) should not receive local anesthesia involving these substances.

To our knowledge, these kinds of data have not been well systematized in a useful data bank like the Japanese Adverse Drug Event Report database of the Pharmaceutical and Medical Devices Agency that are available to the public. To build this kind of a database, individual governments, relevant international medical societies, and/or public organizations should function as a common platform to begin collection and analysis of clinical data, then propose both indications and contraindications, and finally should test the validity of this classification prior to dissemination of the above information to the public. In advance of or in parallel with building the database, we should further develop sensitive but convenient methods for evaluation of topical and systemic conditions.

Several clinicians and researchers, including our group, have repeatedly proposed the necessity of standardized preparation protocols and therapeutic procedures, for example, in terms of timing and dosing [17, 23-25, 43]. However, we recently came to realize that the priority of research should be shifted from standardization of PC preparation and applications to clarification of indications and contraindications for successful PC therapy.

\section{Conclusion}

To date, high-quality RCTs with large numbers of human subjects have not been conducted to evaluate PC therapy in terms of both safety and efficacy regardless of specific fields of regenerative medicine. Contradictory results regarding efficacy have always been attributed to the differences in individual samples and preparation protocols without careful consideration or discussion, while safety has been endorsed seemingly without careful or long-term standardized evaluation. Both clinicians and basic researchers have paid much attention to PC efficacy and have proposed standard preparation protocols (to produce similar PC preparations) and universal terminology of PCs to more precisely compare their clinical outcomes [17]. In previous articles [43, 46], we independently proposed the necessity of standardized protocols for PRF preparation and of terminology based on generic rather than vendor names. In this review article, we continue to emphasize the need for rerecognition of "safety myths" of PC therapy and propose performing a specific blood test in the spirit of GMP-based manufacturing and surveying patients' medical history more carefully than usual prior to PC therapy. We believe that standardization of such a series of preoperative assessments is urgently needed and that this preoperative examination will reduce inappropriate therapeutic use of PCs and simultaneously improve general quality of this modality.

\section{Abbreviations}

PCs: Platelet concentrates

PRP: Platelet-rich plasma

PRF: Platelet-rich fibrin 
MHLW: The Ministry of Health, Labour and Welfare of Japan

CBMP: Cell-based medicinal product

GMP: Good manufacturing practice

EDTA: Ethylenediamine tetra-acetic acid

ACD: Acid-citrate-dextrose

RCT: Randomized controlled trial

SOP: $\quad$ Standard operating procedure

ES cells: Embryonic stem cells

iPS cells: Induced pluripotent stem cells

GVHD: Graft-versus-host disease.

\section{Conflicts of Interest}

The authors declare that they have no conflicts of interest.

\section{References}

[1] M. Sánchez, A. Garate, D. Delgado, and S. Padilla, "Platelet-rich plasma, an adjuvant biological therapy to assist peripheral nerve repair," Neural Regeneration Research, vol. 12, no. 1, pp. 47-52, 2017.

[2] R. S. Dhillon, E. M. Schwarz, and M. D. Maloney, "Plateletrich plasma therapy-future or trend?" Arthritis Research \& Therapy, vol. 14, no. 4, p. 219, 2012.

[3] K. Beitzel, D. Allen, J. Apostolakos et al., "US definitions, current use, and FDA stance on use of platelet-rich plasma in sports medicine," The Journal of Knee Surgery, vol. 28, no. 1, pp. 29-34, 2015.

[4] S. Fiorentino, A. Roffi, G. Filardo, M. Marcacci, and E. Kon, "European definitions, current use, and EMA stance of plateletrich plasma in sports medicine," The Journal of Knee Surgery, vol. 28, no. 1, pp. 51-54, 2015.

[5] E. Anitua, R. Prado, and G. Orive, "Closing regulatory gaps: New ground rules for platelet-rich plasma," Trends in Biotechnology, vol. 33, no. 9, 2015.

[6] E. Anitua, R. Prado, and G. Orive, "A New Regulatory Framework for Platelet-Rich Plasma in Spain," Journal of Knee Surgery, vol. 28, no. 4, pp. 355-356, 2015.

[7] K. Konomi, M. Tobita, K. Kimura, and D. Sato, "New Japanese initiatives on stem cell therapies," Cell Stem Cell, vol. 16, no. 4, pp. 350-352, 2015.

[8] "The Ministry of Health Labour and Welfare of Japan, "Regenerative Medicine,"," 2018, http://www.mhlw.go.jp/stf/ seisakunitsuite/bunya/kenkou_iryou/iryou/saisei_iryou/.

[9] "The Ministry of Health Labour and Welfare of Japan, "Guidance of the Act on Securement of Safety of Regenerative Medicine," 2018, http://www.mhlw.go.jp/file/06-Seisakujouhou10800000-Iseikyoku/0000079192.pdf.

[10] H. Okura and A. Matsuyama, "History of development and regulations for regenerative medicines in Japan," Journal of Stem Cell Research \& Therapy, vol. 07, no. 01, 2017.

[11] M. Tobita, K. Konomi, Y. Torashima, K. Kimura, M. Taoka, and M. Kaminota, "Japan's challenges of translational regenerative medicine: Act on the safety of regenerative medicine," Regenerative Therapy, vol. 4, pp. 78-81, 2016.

[12] C. A. Herberts, M. S. G. Kwa, and H. P. H. Hermsen, "Risk factors in the development of stem cell therapy," Journal of Translational Medicine, vol. 9, article 29, 2011.
[13] R. Haddock, S. Lin-Gibson, and N. Lumelsky, Manufacturing Cell Therapies: The Paradigm Shift in Health Care of This Century, URL https, 2018, https://nam.edu/wp-content/uploads/ 2017/06/Manufacturing-Cell-Therapies.pdf.

[14] N. Metcalf, P. Hourd, and A. Chandra, Quality assurance and GMP in the manufacture of cell-based therapeutics, 2013.

[15] J. Carmen, S. R. Burger, M. McCaman, and J. A. Rowley, "Developing assays to address identity, potency, purity and safety: Cell characterization in cell therapy process development," Journal of Regenerative Medicine, vol. 7, no. 1, pp. 85-100, 2012.

[16] D. K. Gavin, Advanced Topics: Successful Development of Quality Cell and Gene Therapy Products, 2018, 2018, https://www.fda.gov/ downloads/BiologicsBloodVaccines/NewsEvents/UCM251337. pdf.

[17] M. R. De Pascale, L. Sommese, A. Casamassimi, and C. Napoli, "Platelet derivatives in regenerative medicine: an update," Transfusion Medicine Reviews, vol. 29, no. 1, pp. 52-61, 2015.

[18] J. Margolis, "Glass surface and blood coagulation [14]," Nature, vol. 178, no. 4537, pp. 805-806, 1956.

[19] J. Alsousou, A. Ali, K. Willett, and P. Harrison, "The role of platelet-rich plasma in tissue regeneration," Platelets, vol. 24, no. 3, pp. 173-182, 2013.

[20] K. Isobe, T. Watanebe, H. Kawabata et al., "Mechanical and degradation properties of advanced platelet-rich fibrin (A$\mathrm{PRF}$ ), concentrated growth factors (CGF), and platelet-poor plasma-derived fibrin (PPTF)," International Journal of Implant Dentistry, vol. 3, no. 1, 2017.

[21] K. Okuda, T. Kawase, M. Momose et al., "Platelet-rich plasma contains high levels of platelet-derived growth factor and transforming growth factor- $\beta$ and modulates the proliferation of periodontally related cells in vitro," Journal of Periodontology, vol. 74, no. 6, pp. 849-857, 2003.

[22] Y. Kitamura, T. Watanabe, M. Nakamura et al., "Platelet Counts in Insoluble Platelet-Rich Fibrin Clots: A Direct Method for Accurate Determination," Frontiers in Bioengineering and Biotechnology, vol. 6, no. 4, 2018.

[23] L. F. Marques, T. Stessuk, I. C. C. Camargo, N. Sabeh Junior, L. D. Santos, and J. T. Ribeiro-Paes, "Platelet-rich plasma (PRP): Methodological aspects and clinical applications," Platelets, vol. 26, no. 2, pp. 101-113, 2015.

[24] M. J. Mosca and S. A. Rodeo, "Platelet-rich plasma for muscle injuries: game over or time out?" Current Reviews in Musculoskeletal Medicine, vol. 8, no. 2, pp. 145-153, 2015.

[25] A. Roffi, G. Filardo, E. Kon, and M. Marcacci, "Does PRP enhance bone integration with grafts, graft substitutes, or implants? A systematic review, BMC Musculoskeletal Disorders, vol. 14, article 330, 2013.

[26] A. Szczeklik, J. Musiał, A. Undas et al., "Aspirin and thrombinogenesis," Thrombosis Research, vol. 110, no. 5-6, pp. 345-347, 2003.

[27] R. Tadros and S. Shakib, "Warfarin: Indications, risks and drug interactions," Australian Family Physician, vol. 39, no. 7, pp. 476479, 2010.

[28] D. Wardrop and D. Keeling, "The story of the discovery of heparin and warfarin," British Journal of Haematology, vol. 141, no. 6, pp. 757-763, 2008.

[29] K. Schrör, "Aspirin and platelets: the antiplatelet action of aspirin and its role in thrombosis treatment and prophylaxis," Seminars in Thrombosis and Hemostasis, vol. 23, no. 4, pp. 349356, 1997. 
[30] J. H. Kim, H. Y. Bae, and S. Y. Kim, “Clinical marker of platelet hyperreactivity in diabetes mellitus," Diabetes \& Metabolism, vol. 37, no. 6, pp. 423-428, 2013.

[31] P. Kubisz, L. Stanciaková, J. Staško, P. Galajda, and M. Mokáň, "Endothelial and platelet markers in diabetes mellitus type 2," World Journal of Diabetes, vol. 6, no. 3, pp. 423-431, 2015.

[32] B. J. McEwen, "The influence of diet and nutrients on platelet function," Seminars in Thrombosis and Hemostasis, vol. 40, no. 2, pp. 214-226, 2014.

[33] S. Rajaram, "The effect of vegetarian diet, plant foods, and phytochemicals on hemostasis and thrombosis," American Journal of Clinical Nutrition, vol. 78, no. 3, pp. 552S-558S, 2003.

[34] G. Grignani, L. Pacchiarini, M. Zucchella et al., "Effect of mental stress on platelet function in normal subjects and in patients with coronary artery disease," Pathophysiology of Haemostasis and Thrombosis, vol. 22, no. 3, pp. 138-146, 1992.

[35] N. H. Wallen, C. Held, N. Rehnqvist, and P. Hjemdahl, "Effects of mental and physical stress on platelet function in patients with stable angina pectoris and healthy controls," European Heart Journal, vol. 18, no. 5, pp. 807-815, 1997.

[36] O. Naesh, J. T. Friis, and I. Hindberg, "Platelet function in surgical stress," Thrombosis and Haemostasis, vol. 54, pp. 849852, 1985.

[37] P. Koudouovoh-Tripp, "Influence of mental stress on platelet bioactivity," World Journal of Psychiatry, vol. 2, no. 6, p. 134, 2012.

[38] D. J. O'Keeffe and T. P. Baglin, “Travellers' thrombosis and economy class syndrome: Incidence, aetiology and prevention," Clinical \& Laboratory Haematology, vol. 25, no. 5, pp. 277-281, 2003.

[39] F. Graziani, S. Ivanovski, S. Cei, F. Ducci, M. Tonetti, and M. Gabriele, "The in vitro effect of different PRP concentrations on osteoblasts and fibroblasts," Clinical Oral Implants Research, vol. 17, no. 2, pp. 212-219, 2006.

[40] H. Masuki, T. Okudera, T. Watanebe et al., "Growth factor and pro-inflammatory cytokine contents in platelet-rich plasma (PRP), plasma rich in growth factors (PRGF), advanced platelet-rich fibrin (A-PRF), and concentrated growth factors (CGF)," International Journal of Implant Dentistry, vol. 2, no. 1, 2016.

[41] M. Nagata, H. Hoshina, M. Li et al., "A clinical study of alveolar bone tissue engineering with cultured autogenous periosteal cells: coordinated activation of bone formation and resorption," Bone, vol. 50, no. 5, pp. 1123-1129, 2012.

[42] S. Ogawa, H. Hoshina, K. Nakata et al., "High-Resolution ThreeDimensional Computed Tomography Analysis of the Clinical Efficacy of Cultured Autogenous Periosteal Cells in Sinus Lift Bone Grafting," Clinical Implant Dentistry and Related Research, vol. 18, no. 4, pp. 707-716, 2016.

[43] M. Kobayashi, T. Kawase, M. Horimizu, K. Okuda, L. F. Wolff, and $\mathrm{H}$. Yoshie, "A proposed protocol for the standardized preparation of PRF membranes for clinical use," Biologicals, vol. 40, no. 5, pp. 323-329, 2012.

[44] T. Kawase, "Platelet-rich plasma and its derivatives as promising bioactive materials for regenerative medicine: basic principles and concepts underlying recent advances," Odontology, vol. 103, no. 2, pp. 126-135, 2015.

[45] S. Sampson, M. Gerhardt, and B. Mandelbaum, "Platelet rich plasma injection grafts for musculoskeletal injuries: a review,"
Ethics in Science and Environmental Politics, vol. 1, no. 3-4, pp. 165-174, 2008.

[46] T. Kawase and T. Tanaka, "An updated proposal for terminology and classification of platelet-rich fibrin," Regenerative Therapy, vol. 7, pp. 80-81, 2017. 


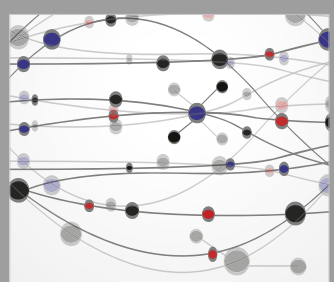

The Scientific World Journal
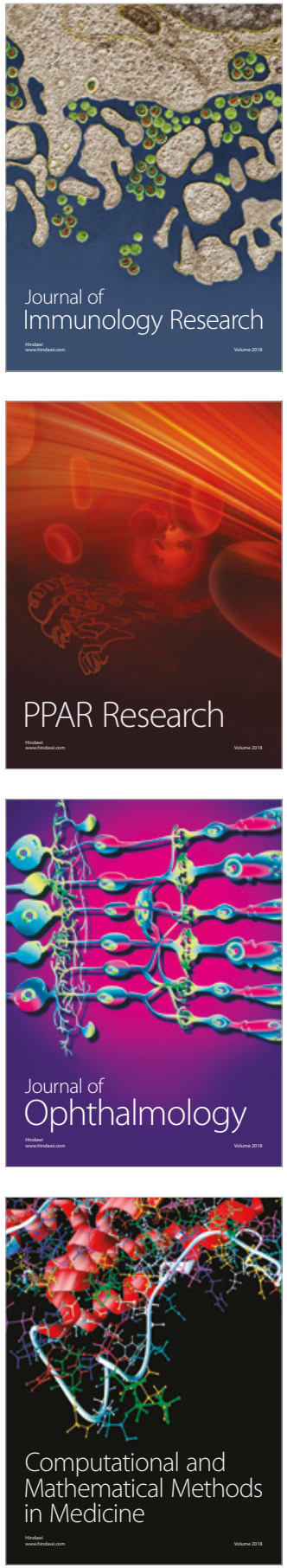

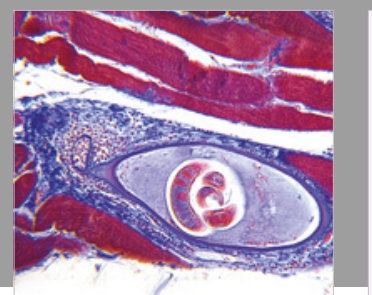

Gastroenterology Research and Practice

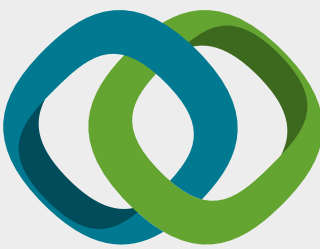

\section{Hindawi}

Submit your manuscripts at

www.hindawi.com
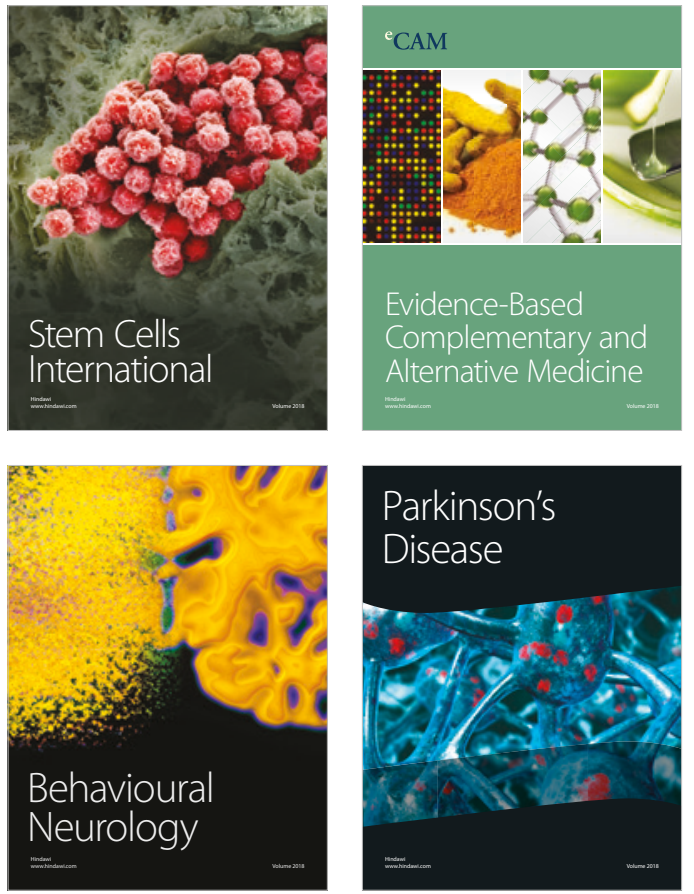

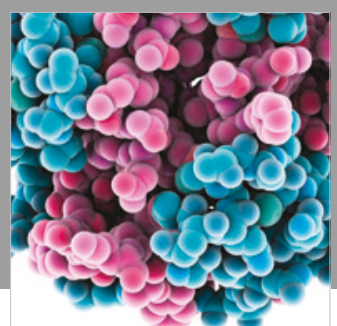

ournal of

Diabetes Research

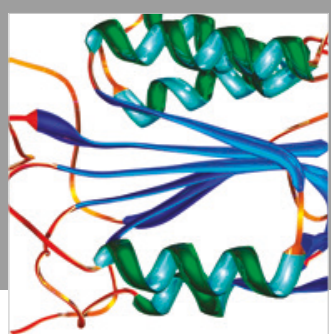

Disease Markers
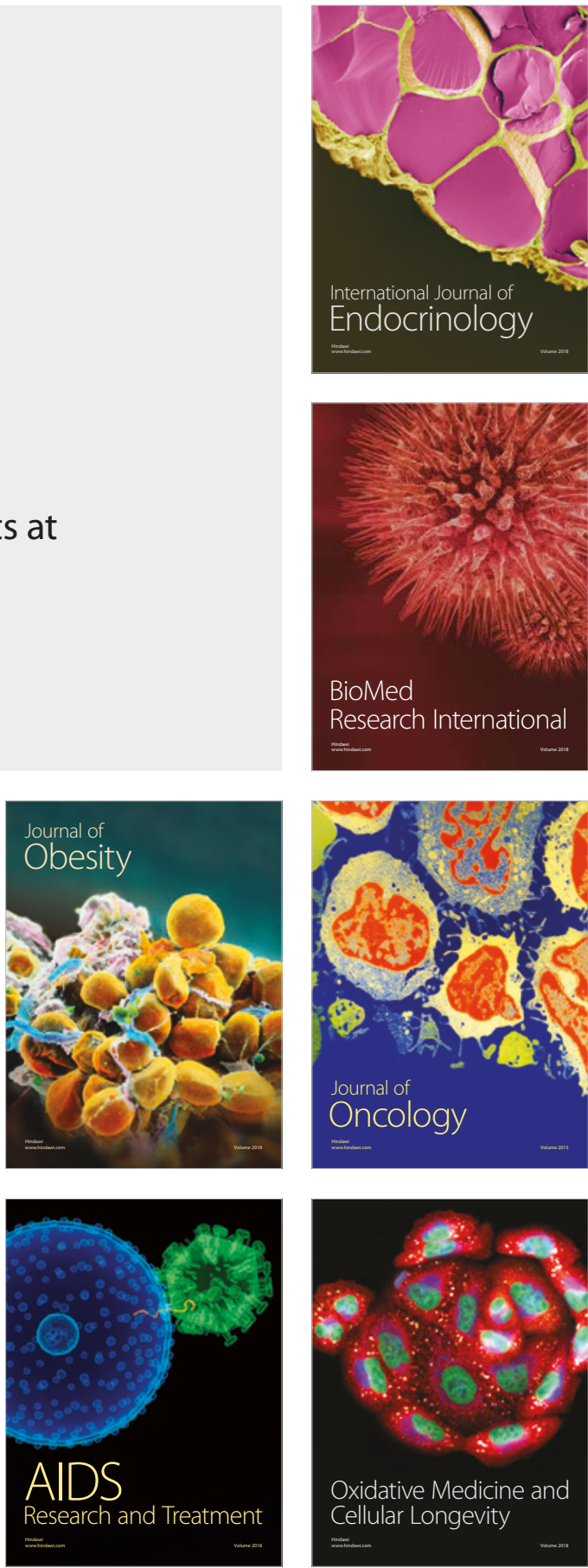